\title{
Expression of latent membrane proteins in Epstein-Barr virus-transformed lymphocytes in vitro
}

\author{
YUNLIAN TANG*, CHUNYAN LUO*, AILAN CHENG, SULI LU, JINHUA XU, TING FU and RUNLIANG GAN \\ Cancer Research Institute, University of South China, Hengyang, Hunan 421001, P.R. China
}

Received August 21, 2013; Accepted April 7, 2014

DOI: $10.3892 / \mathrm{mmr} .2014 .2313$

\begin{abstract}
Infection with Epstein-Barr virus (EBV) induces activation and proliferation of B lymphocytes. Detection of latent membrane protein (LMP)-1 is used to identify the proliferative ability of B cells. However, changes in the expression levels of the three LMPs during EBV-induced B lymphocyte transformation, have not yet been reported. In the present study, the expression levels of LMP-1, LMP-2A and LMP-2B were compared between EBV-transformed B lymphocytes and paired normal lymphocytes. Seven lymphoblast cell lines were established by EBV infection of normal human lymphocytes in vitro. The expression levels of LMP genes and LMP-1 protein were determined using quantitative (q)PCR and western blotting in lymphoblasts and normal lymphocytes, respectively. The expression of $L M P 1$, $L M P-2 A$ and $L M P-2 B$ genes was significantly upregulated in EBV-induced lymphoblasts compared with the normal lymphocytes. The LMP-1 protein level was also significantly increased in EBV-transformed B lymphocytes. Expression of $L M P 1, L M P-2 A$ and $L M P-2 B$ genes was significantly upregulated in EBV-induced lymphoblasts, suggesting LMP genes are important in the transformation of human lymphocytes.
\end{abstract}

\section{Introduction}

Epstein-Barr virus (EBV) is a double-stranded DNA virus of the herpes family, that targets lymphocytes and is closely associated with multiple malignancies, including lymphoma, nasopharyngeal cancer and gastric cancer (1). Epithelial cells, lymphocytes and muscle cells are particularly vulnerable to EBV (2). Similar to other herpes viruses, EBV has the capacity

Correspondence to: Professor Runliang Gan, Cancer Research Institute, University of South China, 28 Chang Sheng Xi Avenue, Hengyang, Hunan 421001, P.R. China

E-mail: gan988@yahoo.com

*Contributed equally

Key words: Epstein-Barr virus, lymphocyte transformation, latent membrane protein-1, latent membrane protein-2A, latent membrane protein-2B to induce lytic and latent infection. Latent EBV infection results in the expression of only minimal numbers of viral proteins, but it is able to bypass host immune surveillance and thus carries a tumorigenic risk. Despite this, cytologists often encounter EBV-associated malignancies in cytological material, but in contrast to other herpes viruses, EBV does not evoke viral cytopathic effects, such as cell merging and necrosis (2). In vitro infection with EBV induces activation and proliferation of human B lymphocytes (3). EBV-specific cytotoxic $\mathrm{CD}^{+} \mathrm{T}$ cells are responsible for the clearance of EBV-infected lymphocytes by recognizing the virus-coded proteins and therefore the majority of EBV carriers are asymptomatic throughout life (3). Expression of LMP-1 protein is associated with the proliferation of B lymphocytes. By contrast, LMP-1 protein is absent in latent infection with EBV type I and IIb, and infected cells have no inherent proliferation capacity (4). Therefore, LMP-1 expression is used to determine the proliferative ability of B lymphocytes.

Latent membrane proteins (LMPs) have three subtypes, LMP-1, LMP-2A and LMP-2B. Expression of the three genes during EBV-induced transformation of human B lymphocytes has not been investigated. In the present study, the expression of $L M P-1, L M P-2 A$ and $L M P-2 B$ genes in EBV-induced lymphoblasts and paired normal lymphocytes was compared to elucidate its significance during lymphocyte transformation.

\section{Materials and methods}

Blood samples. Peripheral blood was collected from seven healthy volunteers. The study was approved by the Ethics Committee of University of South China, Hengyang, China. Written informed consent was obtained from the patients. EBV infection status was detected with an ELISA kit (ADL Embedded Solutions Inc., San Diego, CA, USA) using the anti-EBV-VCA IgG antibody (ADL Embedded Solutions Inc.). DNA was extracted from the whole blood samples and the LMP-1 gene sequence ( 82 bp, GI: 896226) was expanded using polymerase chain reaction (PCR). The upstream primer of the LMP-1 gene was: 5'-CTG CTC ATC GCT CTC TGG AA-3' and the downstream primer was: 5'-AGA CAA GTA AGC ACC CGA AGA TG-3'. The PCR included 30 cycles of $94^{\circ} \mathrm{C}$ for $4 \mathrm{~min}, 94^{\circ} \mathrm{C}$ for $30 \mathrm{sec}, 52^{\circ} \mathrm{C}$ for $30 \mathrm{sec}$ and $72^{\circ} \mathrm{C}$ for $30 \mathrm{sec}$, and a final extension at $72^{\circ} \mathrm{C}$ for $5 \mathrm{~min}$. The PCR products were separated on $2 \%$ agarose gels. The results demonstrated EBV latency in the seven blood samples. 
Table I. Gene-specific PCR primer sequences (5'-3').

\begin{tabular}{lllr}
\hline Gene name & \multicolumn{1}{c}{ Forward primer } & \multicolumn{1}{c}{ Reverse primer } & Size $(\mathrm{bp})$ \\
\hline LMP-1 & CTGCTCATCGCTCTCTGGAA & AGACAAGTAAGCACCCGAAGATG & 82 \\
LMP-2A & CGTCACTCGGACTATCAACCAC & CTTCCTCTGCCCGCTTCTT & 149 \\
LMP-2B & CGCCGTTTGACTGTTTGTG & AGCAGCAGCGTCATGGAA & 125 \\
GAPDH & GCACCGTCAAGGCTGAGAAC & TGGTGAAGACGCCAGTGGA & 138
\end{tabular}

PCR, polymerase chain reaction; LMP, latent membrane protein.

Isolation of lymphocytes. Peripheral blood $(50 \mathrm{ml})$ was collected from seven healthy volunteers. Normal PBLs were separated from fresh peripheral blood samples using human lymphocyte separation medium (catalogue no. LTS1077; Tian Jin Hao Yang Biological Manufacture Co., Ltd., Tianjin, China) and washed twice with RPMI-1640.

Isolation of EBV. The B95-8 marmoset cell line was kindly provided by the Cancer Research Institute, Central South University, Changsha, China and used as a source of EBV. The culture medium of B95-8 cells was replenished for the final time. The cell density was adjusted to $10^{6}-10^{7} / \mathrm{ml}$. The cells were starved for 10 days at $37^{\circ} \mathrm{C}$ in $5 \% \mathrm{CO}_{2}$ and were then centrifuged at $3,700 \times \mathrm{g}$ at $4^{\circ} \mathrm{C}$ for $30 \mathrm{~min}$. The supernatant was passed through a $0.45-\mu \mathrm{m}$ filter and stored at $-80^{\circ} \mathrm{C}$.

Preparation of lymphoblasts. A total of $2 \times 10^{6}-3 \times 10^{6}$ lymphocytes were suspended in $2 \mathrm{ml}$ RPMI-1640 culture medium supplemented with $25 \%$ fetal bovine serum (Gibco, Sydney, Australia) and $2 \mu \mathrm{g} / \mathrm{ml}$ cyclosporine A (Sandoz, Basel, Switzerland). The cells were transferred into a 24-well plate and incubated at $37^{\circ} \mathrm{C}$ with $5 \% \mathrm{CO}_{2}$ for one week. Subsequently, the lymphocytes were induced into lymphoblasts that were enlarged and exhibited cell clustering. The culture medium was replenished every 3-4 days and $\sim 4$ weeks later the cells were transferred into 25 -ml flasks for further culture.

Quantatitive (q)PCR. Total RNA was extracted from lymphoblasts, untreated lymphocytes and B95-8 cells using TRIzol reagent (Invitrogen Life Technologies, Carlsbad, CA, USA). A total of $2 \times 10^{6}$ cells were used for RNA extraction. The purity and integrity of the extracted RNA were examined using electrophoresis. RNA was reverse transcribed into cDNA (Promega Corporation, Madison, WI, USA) according to the manufacturer's instructions. The primers used in the qPCR are listed in Table I. The B95-8 cells that expressed the EBV genome were used as a positive control to establish the standard curve for qPCR. Each test was repeated twice.

Western blotting. Total protein was extracted from the cells using SDS lysis buffer (Beyotime, Shanghai, China) and quantified using an Enhanced Bicinchoninic Acid Protein Assay kit (Beyotime). The protein was denatured at $95^{\circ} \mathrm{C}$ for $10 \mathrm{~min}$ and then $50 \mu \mathrm{g}$ was separated in $8-12 \%$ SDS-PAGE and transferred onto a nitrocellulose membrane (Beyotime). The membrane was blocked with $5 \%$ skimmed milk in Tris buffer $(25 \mathrm{mM}$

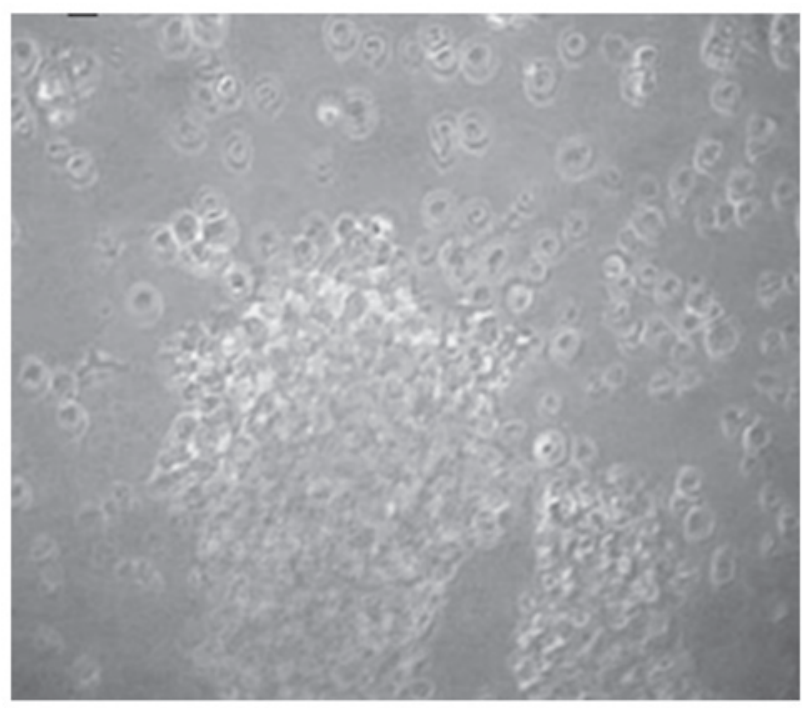

Figure 1. Epstein-Barr virus-induced lymphoblasts were round or oval, and the cells were clustered and suspended in the medium (magnification, x200).

Tris- $\mathrm{HCl}, 150 \mathrm{mM} \mathrm{NaCl}$ and $0.05 \%$ Tween-20, $\mathrm{pH} 7.5)$. Mouse anti-LMP-1 monoclonal antibody (1:200; DakoCytomation, Glostrup, Denmark) and mouse anti- $\beta$-actin monoclonal antibody (1:1000; ComWin, Beijing, China) were added and the membranes were incubated at $4^{\circ} \mathrm{C}$ overnight. Goat anti-mouse IgG (1:1000; ComWin) was used as a secondary antibody, incubated at room temperature for $2 \mathrm{~h}$. Each test was performed in triplicate.

Statistical analysis. All the data are expressed as the mean \pm standard deviation. Data were analyzed using one-way analysis of variance. $\mathrm{P}<0.05$ was considered to indicate a statistically significant difference.

\section{Results}

Lymphoblast morphology. EBV-transformed lymphoblasts (LCLs) were round or oval and enlarged in size compared with the normal lymphocytes (Fig. 1).

Expression of LMP genes. Melting curve analysis demonstrated only one specific peak for LMP-1, LMP-2A and LMP-2B, indicating high specificity of the qPCR (Fig. 2).

PCR products of LMP genes were separated on $2 \%$ agarose gels, which revealed well-separated bands of the predicted sizes (Fig. 3). 
Table II. Expression levels of LMP genes as determined by qPCR

\begin{tabular}{lcrr}
\hline Cell type & LMP1 \pm SD & LMP-2A \pm SD & LMP-2B \pm SD \\
\hline Normal lymphocytes & $2.414 \times 10^{-4} \pm 1.080 \times 10^{-4}$ & $2.97 \times 10^{-4} \pm 1.64 \times 10^{-4}$ & $6.40 \times 10^{-5} \pm 3.04 \times 10^{-5}$ \\
Induced lymphoblasts & $2.082 \times 10^{-1} \pm 6.120 \times 10^{-2}$ & $5.235 \times 10^{-1} \pm 2.37 \times 10^{-1}$ & $5.765 \pm 2.914$ \\
\hline
\end{tabular}

$\mathrm{P}<0.01$ for LMP-1, $\mathrm{P}<0.05$ for LMP-2A and LMP-2B; comparison between induced lymphoblasts and normal lymphocytes (n=7). LMP, latent membrane protein; qPCR, quantatitive polymerase chain reaction; $\mathrm{SD}$, standard deviation.

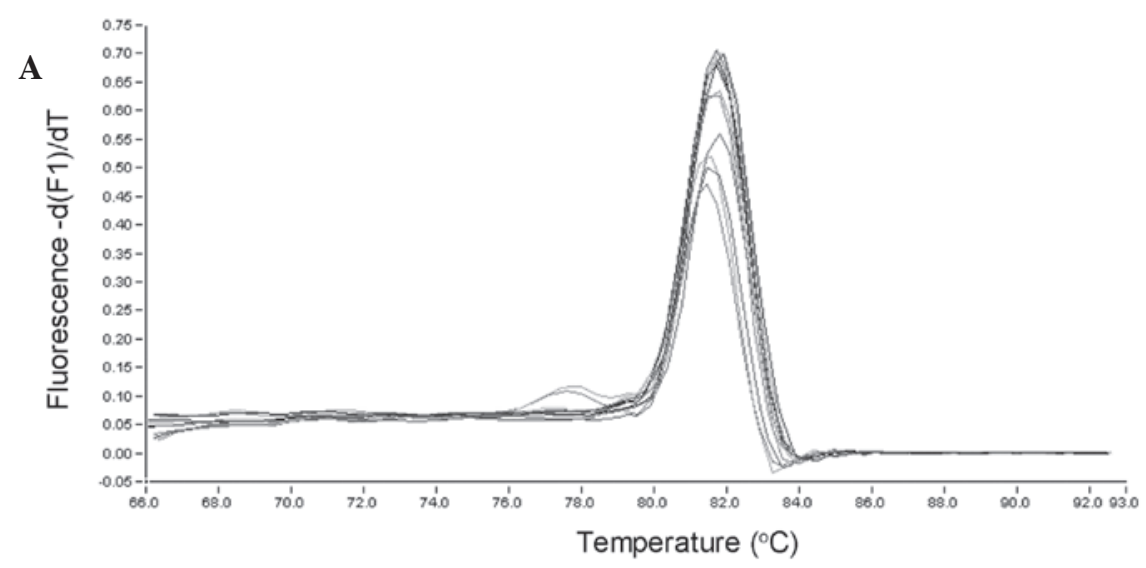

Digital Filter: Enabled Calculation Method: Polynomial

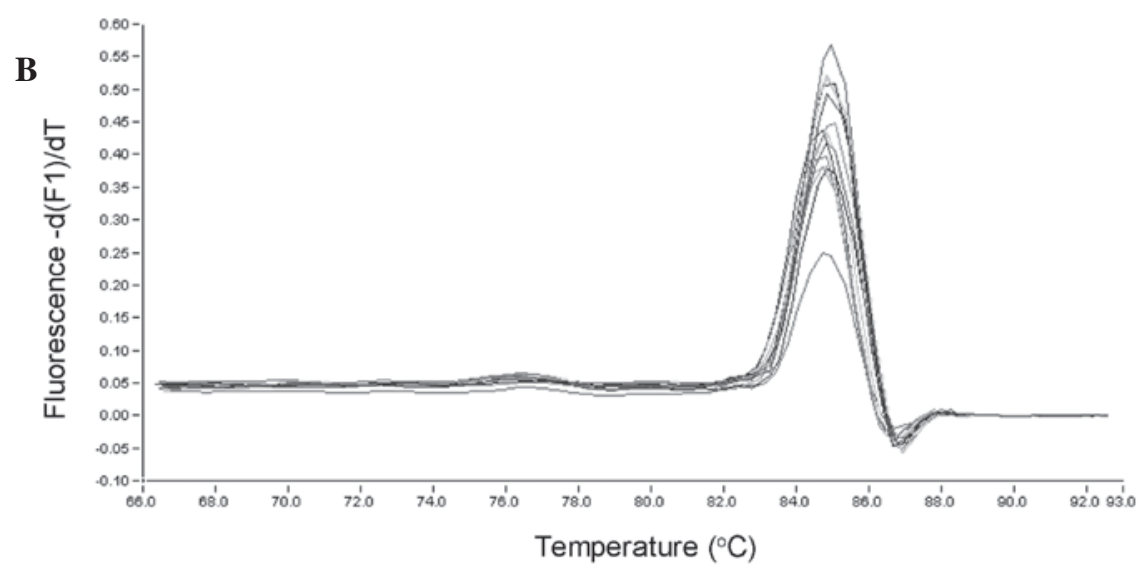

Digital Filter: Enabled Calculation Method: Polynomial

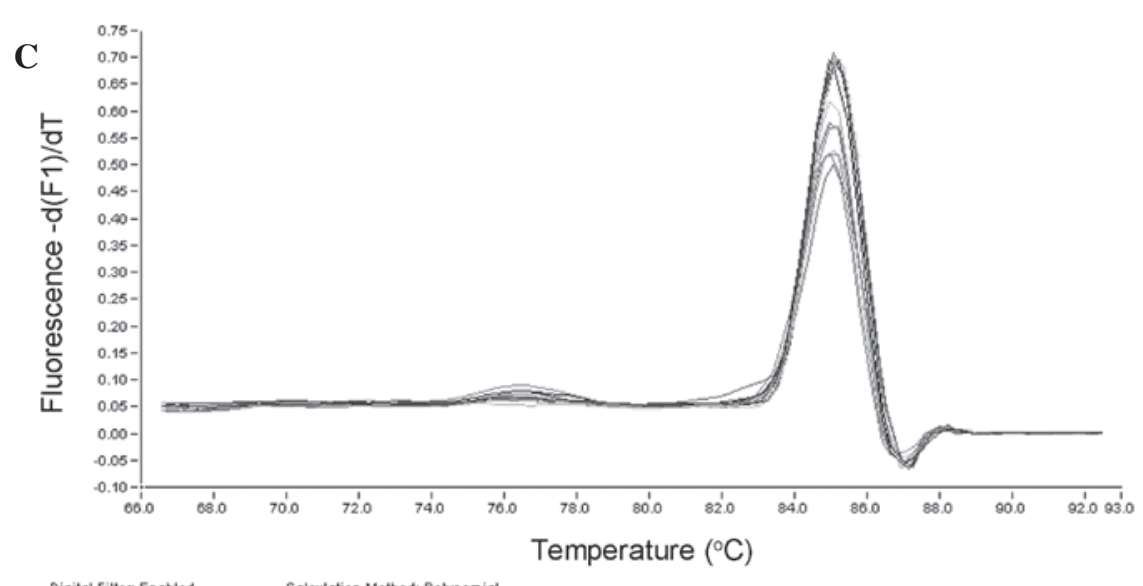

Figure 2. Melting curves of quantitative polymerase chain reaction detecting the expression of (A) LMP-1; (B) LMP-2A and (C) LMP-2B, all of which had one specific peak each. LMP, latent membrane protein. 

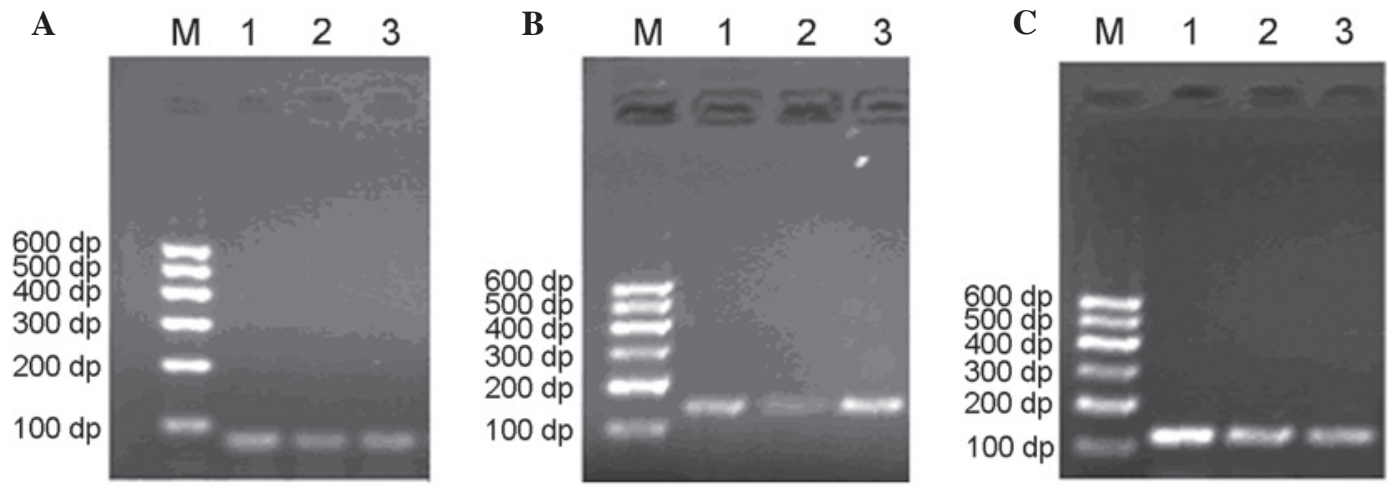

Figure 3. Polymerase chain reaction products of LMP genes on agarose gels. Lane 1, positive control (B95-8 cells); lane 2, paired untreated lymphocytes; lane 3, lymphoblasts. (A) LMP-1, 82 bp; (B) LMP-2A, 149 bp and (C) LMP-2B, 125 bp. LMP, latent membrane protein; M, marker.
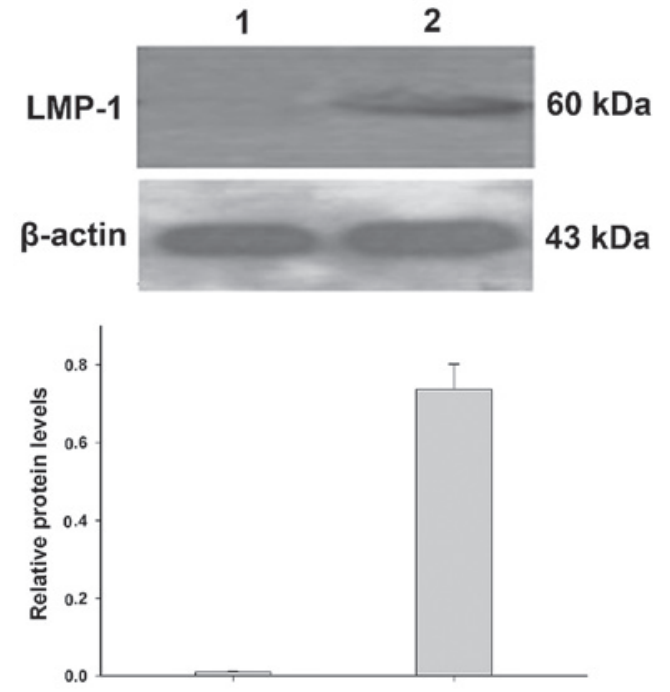

Figure 4. LMP-1 protein levels in EBV-transformed lymphoblasts and normal lymphocytes. Western blotting revealed that LMP-1 protein levels were significantly increased in EBV-induced lymphoblasts compared with that in normal lymphocytes $(\mathrm{P}<0.01)$. Lane 1 , normal lymphocytes; lane 2, EBV-transformed lymphoblasts. LMP, latent membrane protein; EBV, Epstein-Barr virus.

Expression levels of LMP-1, LMP-2A and LMP-2B in EBV-transformed lymphoblasts were 863-fold $(\mathrm{P}<0.01)$, 1,763 -fold $(\mathrm{P}<0.05)$ and 90,078 -fold $(\mathrm{P}<0.05)$ of that in untreated lymphocytes, respectively (Table II).

Detection of LMP-1 protein levels. Western blotting demonstrated that LMP-1 protein levels were significantly increased in EBV-transformed lymphoblasts compared with that in the normal lymphocytes $(\mathrm{P}<0.05$; Fig. 4), which is consistent with the results of qPCR.

\section{Discussion}

EBV is considered to be associated with several malignancies, including Hodgkin's lymphoma, NK-T cell lymphoma, Burkitt's lymphoma and nasopharyngeal cancer (5). Latent EBV infection induces the expression of three LMP proteins, LMP-1, LMP-2A and LMP-2B (6). LMP-1 is the major factor that induces transformation and tumorigenesis of $\mathrm{B}$ cells (7) and mimics the constitutionally activated receptor of tumor necrosis factor (TNF), CD40. LMP-1, via this TNF receptor, regulates cell proliferation and death (8) and is thus important in cell growth, differentiation and apoptosis. LMP-2A and LMP-2B are two isoforms of the LMP-2 protein expressed in $B$ cells with latent EBV infection (9). LMP-2 proteins promote the development and progression of tumors (10). LMP-2A in particular, has been demonstrated to protect B cells from various proapoptotic mechanisms (11).

EBV latency in healthy carriers is usually asymptomatic. In the present study, it was identified that expression of LMP mRNA in normal lymphocytes was low at $10^{-4}-10^{-5}$, which was significantly upregulated in the EBV-induced lymphoblasts at $2 \times 10^{-2}$. The expression levels of LMP mRNA were determined using qPCR.

The expression of the $L M P-1$ gene was significantly increased in the EBV-transformed lymphoblasts at the mRNA (863-fold; $\mathrm{P}<0.01)$ and protein levels compared with that in normal lymphocytes. Rasul et al (3) cultured mononuclear cells with the supernatant of B95-8 cells for $1.5 \mathrm{~h}$ to infect B cells with EBV. By using immunostaining and western blotting following seven days in culture, it was identified that the B cells expressing LMP-1 protein were mostly positive for Ki-67, while those not expressing LMP-1 protein demonstrated a weak Ki-67 expression and were non-proliferative. Only EBV nuclear antigen (EBNA)-1 was expressed in cells with type I EBV latency, and these cells were nonproliferative and revealed a resting $\mathrm{B}$ cell phenotype, which was also observed in the control memory B cells in healthy individuals. B cells with type IIb EBV latency expressed all the EBNAs but not LMP-1, and these cells were also non-proliferative (4). Therefore, it was considered that the expression of LMP-1 is critical in the proliferation and transformation of B cells (12). EBV-induced immortal lymphoblasts promoted lymphoma genesis (13). Increased expression of LMP-1 mRNA and protein promoted the proliferation of $\mathrm{NK} / \mathrm{T}$ lymphoma cells (14). LMP-1 expression is also a typical feature of R/S cells in Hodgkin's lymphoma and improves the survival and proliferation of R/S cells by altering the cellular phenotype and interacting with the surrounding microenvironment (3).

The mechanisms underlying the proliferation and transformation of B cells promoted by LMP-1 may include the activation of cell signaling pathways and the increase in cell cycle activators. It has been demonstrated that LMP-1 activates 
$\beta$-catenin via the phosphatidylinositol 3-kinase/AKT pathway, thus promoting the proliferation of EBV-infected B cells (7). LMP-1 regulates the expression of death-associated protein kinase 1 and activates nuclear factor $(\mathrm{NF})-\kappa \mathrm{B}$ signaling in LCL cells, thus upregulating the MHC class I antigen processing pathway (8). LMP-1 also induces $\mathrm{CD} 8^{+} \mathrm{T}$ cell reaction and bypasses immune surveillance (15). The expression of LMP1-induced protein (LMPIP) is increased in EBV-infected peripheral lymphocytes and LMP-1-transfected 293 cells. Nasopharyngeal carcinoma cells overexpressing LMPIP demonstrated a decrease in G1 phase cells and an increase in sub-G1 phase cells, accompanied by an increase in cell cycle activators cyclin D1 and cyclin-dependent kinase 4 (16). It has also been revealed that EBV promotes epithelial tumorigenesis by downregulating microRNA-203 via LMP-1 (17).

In the present study, it was identified that expression of LMP-2A in EBV-transformed lymphoblasts was 1,763-fold $(\mathrm{P}<0.05)$ of that in untreated lymphocytes, suggesting that LMP-2A is important in B cell transformation. LMP-2A maintains the persistence of EBV infection by inhibiting the activation of B cells. LMP-2A mRNA is consistently expressed in primary and metastasized nasopharyngeal cancer, suggesting that LMP-2A has an initiating role in EBV-associated malignancy (5). LMP-2A regulates the expression of tumor necrosis receptor-associated factor 2 and thus modulates LMP-1-induced activation of the NF- $\kappa \mathrm{B}$ pathway, finally preventing the apoptosis of lymphoma cells $(18,19)$. LMP-2A induces expression of $\Delta \mathrm{Np} 63 \alpha$ and regulates the proliferation, transformation and differentiation of epithelial cells, which may promote the growth of malignant tumors (20). It has also been identified that LMP-2A promotes malignant transformation by enhancing the cell cycle, inhibiting apoptosis and regulating LMP-1 expression (11). These results suggest that LMP-2A is important in the processes of transformation and tumorigenesis.

It was also identified that the expression of LMP-2B in EBV-transformed lymphoblasts was 90,078-fold $(\mathrm{P}<0.05)$ greater than that in the untreated lymphocytes. LMP-2B modulates the activity of LMP-2A during the transformation of $B$ cells and maintains persistent EBV latency together with LMP-2A (21). LMP-2B inhibits LMP-2A and prevents the potential lytic viral replication of EBV. In addition, upregulated expression of LMP-2B promotes the progression from EBV latency to replicative infection (9).

In conclusion, LMP-2A and LMP-1 promote the proliferation, survival and transformation of B cells. LMP-1 and LMP-2 are frequently expressed in EBV-associated lymphoma and epithelial carcinoma, and therefore may promote tumor progression.

\section{Acknowledgements}

This study was supported by the National Natural Science Foundation of China (grant nos. 81272182 and 81372134) and the Construct Program of the Key Discipline in Hunan Province (grant no. 2011-76).

\section{References}

1. Lan K, Verma SC, Murakami M, Bajaj B and Robertson ES: Epstein-Barr Virus (EBV): infection, propagation, quantitation, and storage. Curr Protoc Microbiol Chapter 14: Unit 14E 12, 2007.

2. Michelow P, Wright $C$ and Pantanowitz L: A review of the cytomorphology of Epstein-Barr virus-associated malignancies. Acta Cytol 56: 1-14, 2012.

3. Rasul AE, Nagy N, Sohlberg E, Ádori M, Claesson HE, Klein G and Klein E: Simultaneous detection of the two main proliferation driving EBV encoded proteins, EBNA-2 and LMP-1 in single B cells. J Immunol Methods 385: 60-70, 2012.

4. Klein E, Kis LL and Klein G: Epstein-Barr virus infection in humans: from harmless to life endangering virus-lymphocyte interactions. Oncogene 26: 1297-1305, 2007.

5. Pang MF, Lin KW and Peh SC: The signaling pathways of Epstein-Barr virus-encoded latent membrane protein 2A (LMP2A) in latency and cancer. Cell Mol Biol Lett 14: 222-247, 2009.

6. Kanegane H, Yachie A, Miyawaki T and Tosato G: EBV-NK cells interactions and lymphoproliferative disorders. Leuk Lymphoma 29: 491-498, 1998.

7. Tomita M, Dewan MZ, Yamamoto N, Kikuchi A and Mori N: Epstein-Barr virus-encoded latent membrane protein 1 activates beta-catenin signaling in B lymphocytes. Cancer Sci 100: 807-812, 2009.

8. Lee CW, Leu SJ, Tzeng RY, et al: Latent membrane protein 1 of Epstein-Barr virus regulates death-associated protein kinase 1 in lymphoblastoid cell line. Virology 413: 19-25, 2011.

9. Rechsteiner MP, Berger C, Zauner L, et al: Latent membrane protein $2 \mathrm{~B}$ regulates susceptibility to induction of lytic Epstein-Barr virus infection. J Virol 82: 1739-1747, 2008.

10. Shair KH, Bendt KM, Edwards RH, Nielsen JN, Moore DT and Raab-Traub N: Epstein-Barr virus-encoded latent membrane protein 1 (LMP1) and LMP2A function cooperatively to promote carcinoma development in a mouse carcinogenesis model. J Virol 86: 5352-5365, 2012.

11. Bultema R, Longnecker $\mathrm{R}$ and Swanson-Mungerson $\mathrm{M}$ : Epstein-Barr virus LMP2A accelerates MYC-induced lymphomagenesis. Oncogene 28: 1471-1476, 2009.

12. Zhang B, Kracker S, Yasuda T, et al: Immune surveillance and therapy of lymphomas driven by Epstein-Barr virus protein LMP1 in a mouse model. Cell 148: 739-751, 2012.

13. Dellis O, Arbabian A, Papp B, Rowe M, Joab I and Chomienne C: Epstein-Barr virus latent membrane protein 1 increases calcium influx through store-operated channels in B lymphoid cells. J Biol Chem 286: 18583-18592, 2011.

14. Ramakrishnan R, Donahue H, Garcia D, Tan J, Shimizu N, Rice AP and Ling PD: Epstein-Barr virus BART9 miRNA modulates LMP1 levels and affects growth rate of nasal NK T cell lymphomas. PLoS One 6: e27271, 2011.

15. Yoshizaki T: A novel immune evasion mechanism of LMP-1, an EBV-primary oncogene, in nasopharyngeal carcinoma. Adv Otorhinolaryngol 72: 157-159, 2011.

16. Wang LT, Lin CS, Chai CY, Liu KY, Chen JY and Hsu SH: Functional interaction of Ugene and EBV infection mediates tumorigenic effects. Oncogene 30: 2921-2932, 2011.

17. Yu H, Lu J, Zuo L, et al: Epstein-Barr virus downregulates microRNA 203 through the oncoprotein latent membrane protein 1: a contribution to increased tumor incidence in epithelial cells. J Virol 86: 3088-3099, 2012.

18. Guasparri I, Bubman D and Cesarman E: EBV LMP2A affects LMP1-mediated NF-kappaB signaling and survival of lymphoma cells by regulating TRAF2 expression. Blood 111: 3813-3820, 2008.

19. Vrazo AC, Chauchard M, Raab-Traub N and Longnecker R: Epstein-Barr virus LMP2A reduces hyperactivation induced by LMP1 to restore normal B cell phenotype in transgenic mice. PLoS Pathog 8: e1002662, 2012.

20. Fotheringham JA, Mazzucca S and Raab-Traub N: Epstein-Barr virus latent membrane protein-2A-induced DeltaNp63alpha expression is associated with impaired epithelial-cell differentiation. Oncogene 29: 4287-4296, 2010.

21. Rechsteiner MP, Bernasconi M, Berger C and Nadal D: Role of latent membrane protein 2 isoforms in Epstein-Barr virus latency. Trends Microbiol 16: 520-527, 2008. 